\title{
A RABBIT HUMERUS MODEL OF PLATING AND NAILING OSTEOSYNTHESIS WITH AND WITHOUT STAPHYLOCOCCUS AUREUS OSTEOMYELITIS
}

\author{
D. Arens, M. Wilke, L. Calabro, S. Hackl, S. Zeiter, I. Zderic, R.G. Richards and T.F. Moriarty*
}

AO Research Institute, Davos, Switzerland.

\begin{abstract}
The local mechanical environment at a fracture is known to influence biological factors such as callus formation, immune cell recruitment and susceptibility to infection. Infection models incorporating a fracture are therefore required to evaluate prevention and treatment of infection after osteosynthesis. The aim of this study was to create humane, standardised and repeatable preclinical models of implant-related bone infection after osteosynthesis in the rabbit humerus.

Custom-designed interlocked intramedullary nails and commercially available locking plates were subjected to biomechanical evaluation in cadaveric rabbit humeri; a 10 -week in vivo healing study; a dose response study with Staphylococcus aureus over 4 weeks; and finally, a longterm infection of 10 weeks in the plate model. Outcome measures included biomechanical testing, radiography, histology, haematology and quantitative bacteriology.

Both implants offered similar biomechanical stability in cadaveric bones, and when applied in the in vivo study, resulted in complete radiographic and histological healing and osteotomy closure within 10-weeks. As expected in the infection study, higher bacterial doses led to an increasing infection rate. In both infected groups, there was a complete lack of osteotomy closure at 4 weeks. C-reactive protein (CRP), lymphocyte: granulocyte ratio and weight loss were increased in infected animals receiving IM nails in comparison with non-inoculated equivalents, although this was less evident in the plate group. In the 10-week infection group, healing does not occur in the plated rabbits.

We have successfully developed a rabbit model that is suitable for further studies, particularly those looking into preventative strategies for post-traumatic implant-related osteomyelitis.
\end{abstract}

Keywords: Osteomyelitis; fracture; healing; Intramedullary nail; Locking compression plate; Staphylococcus aureus.

*Address for correspondence:

T F Moriarty

AO Research Institute Davos, AO Foundation

Clavadelerstrasse 8

CH 7270 Davos Platz, Switzerland

Telephone number: +41814142397

E-mail: fintan.moriarty@aofoundation.org

\section{Introduction}

An understanding of the impact of biomechanical stability on fracture healing has evolved over the last half century. The availability of 'biological' fixation options such as closed nailing and minimally invasive bridge plating allows the surgeon to modify the mechanical stability of the fixation construct to suit the fracture pattern encountered (Perren, 2002). The local mechanical environment at a fracture is known to influence biological factors such as callus formation, oxygen tension and immune cell recruitment (Claes et al., 2002; Hankemeier et al., 2001). Importantly, these factors are also implicated in susceptibility to infection, and fixation stability has therefore unsurprisingly been shown to impact on implant-associated infection in animal models (Worlock et al., 1994).

Animal models are mandated in the evaluation of new orthopaedic technologies and treatment modalities, due to the prohibitive logistics of large well-controlled clinical trials. There is, however, an ethical imperative to minimise undue burden upon experimental animals, and at the same time maximise translation potential of any preclinical in vivo trial (Reifenrath et al., 2014). Our understanding of the relevance of fixation stability to infection and healing outcomes behoves animal models that mimic modern clinical fixation constructs. Rabbits have been extensively used in osteomyelitis research (Lazzarini et al., 2006; Metsemakers et al., 2015; Reizner et al., 2014) and methods for creating repeatable localised infection have been established. Unfortunately, the vast majority of rabbit models of implant related osteomyelitis tend to avoid bone fracture, (Alt et al., 2006; Gahukamble et al., 2014; Moojen et al., 2009; Moriarty et al., 2009; Norden, 1970; Odekerken et al., 2014; Smeltzer et al., 1997). Therefore, the biomechanical forces of any implant is limited to potential stress shielding by the implant, but no inter-fragmentary mechanical forces are present. These models are thus unable to evaluate the effect of infection on the course of bone healing. Of the few studies that have described fracture models in rabbits, high exclusion rates or clinically unrealistic implant systems have been described (Fei et al., 2010; Worlock et al., 1994). Fracture models in small (Penn-Barwell et al., 2014) and large (Stewart et al., 2012) animals have emerged in recent years. However, reproducible model in rabbits have been absent.

The aim of this study was to create humane, standardised and repeatable small animal models of fracture fixation associated osteomyelitis in the rabbit. To this end, we have created two humeral osteotomy models, fixed with either a locked plate or a custom designed interlocked intramedullary nail. After confirmation that the implants 
allow full healing in a non-inoculated situation by ten weeks, both models were inoculated with Staphylococcus aureus in a dose response manner to provide implant related osteomyelitis models for both fixation techniques. The infection study lasted four weeks in both models representing an acute infection. The plate model was then selected for a long-term infection study of ten weeks to determine if the bone heals or leads to non-union in the presence of infection. The model is thus proposed as a suitable model for future studies into prevention of posttraumatic implant associated bone infection.

\section{Materials and Methods}

\section{Study design}

In order to develop and test candidate implant constructs, this study progressed through a step-wise approach with the first step being cadaveric testing of both implants to determine suitability for the in vivo study. This was followed by an in vivo proof of principle in a healing study, which was set to run until complete osteotomy healing in order to prove both constructs enable complete healing (10 weeks duration with plates and nails). Next, a dose-response infection study was performed for both plates and nails with inoculation of a human clinical isolate of $S$. aureus, which, as an agent of acute infection, was allowed to progress for four weeks only. Finally, a longterm infection group was performed with a plate group at 10 weeks to establish a model of chronic infection and determine if the bone heals in the presence of infection. In all cases, infection was diagnosed as culture positive animals or histological evidence of infection. The study design and animal numbers used at each stage are shown in Table 1.

\section{Implant design and manufacture}

The intramedullary (IM) nail used in this study is a custom designed rabbit humeral nail made from electropolished Stainless Steel (EPSS, Fig. 1 available from RISystem AG, Davos, Switzerland). The nail is $55 \mathrm{~mm}$ in length, with four interlocking boltholes available. A minor modification of the nail design occurred upon completion of the healing phase, whereby the threaded tip of the nail was shortened by $8 \mathrm{~mm}$ to ensure ease of positioning in the bone. This change simplifies implant placement but does not influence

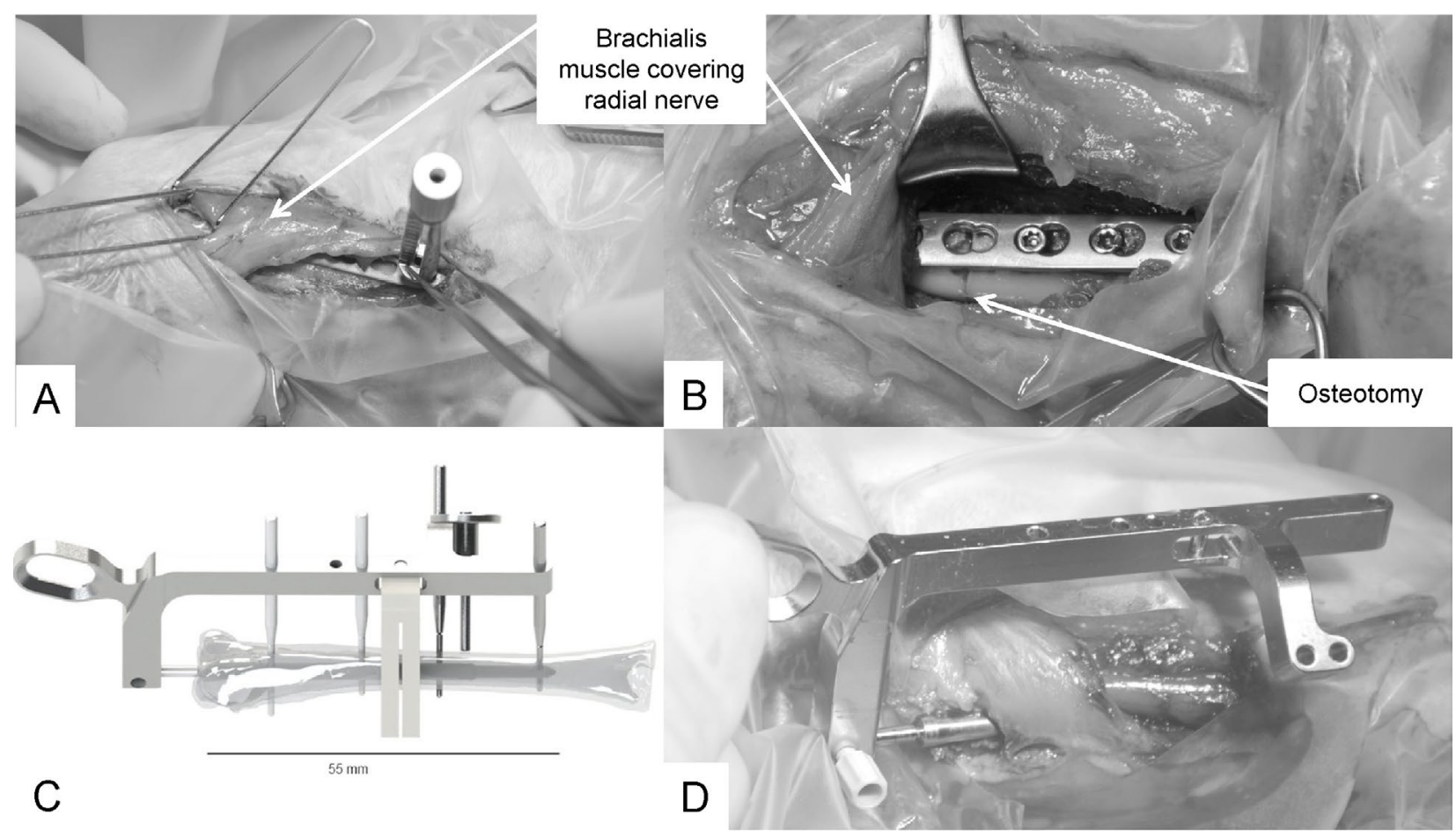

Fig. 1. Images of key features of the plating and nailing models of osteotomy fixation in the rabbit humerus. A \& B, intraoperative image of rabbit receiving plate: Note in $\mathbf{A}$, the brachialis muscle lies above the plate and protects the radial nerve. $\mathbf{B}$, the osteotomy is cut with continual protection of both the muscle and nerve. $\mathbf{C} \& \mathbf{D}$ : Image of nail and aiming device: $\mathbf{C}$, computer generated image of the IM nail showing the entry point of the nail and positioning of the four interlocking bolts (nail length $55 \mathrm{~mm}$ ). The nail is positioned with the aiming device, which is also used for pre-drilling, bolt placement and performance of the osteotomy (image courtesy of RISystem AG). D, intraoperative image shows positioning of the nail. Note: entry point obscured by adjacent musculature.

Table 1. Overview of study design and group sizes for the cadaveric mechanical testing, and in vivo healing and infection studies.

\begin{tabular}{|l|c|c|c|c|c|c|c|c|c|c|}
\hline \multicolumn{2}{|c|}{ Biomechanical testing (cadaver) } & Healing Study (10 weeks) & \multicolumn{5}{|c|}{ Infection (4 weeks) } & Infection (10 weeks) \\
\hline & Test & Control & Not Inoculated & Total & $10^{2}$ & $10^{3}$ & $10^{4}$ & $10^{5}$ & $10^{6}$ & $10^{6}$ \\
\hline Plate & 6 & 6 & 8 & 17 & - & 3 & 6 & 6 & 2 & 8 \\
\hline Nail & 6 & 6 & 8 & 20 & 2 & 4 & 6 & 5 & 3 & - \\
\hline
\end{tabular}


the fixation provided by the nail in any way. Two EPSS bolts were placed proximal and two distal to the intended osteotomy site. Three of the interlocking bolts were positioned in a parallel lateromedial orientation and the fourth was placed at an angle of $45^{\circ}$ to the other three bolts in a craniolateral to caudomedial orientation. Bolts of 10 and $14 \mathrm{~mm}$ in length were available and selected according to intraoperative measurements. The plating constructs used in this study were commercially available $49 \mathrm{~mm}$, 7-hole, EPSS Synthes Veterinary Locking Compression Plates (Synthes, Switzerland). Each plate was fixed with six $2 \mathrm{~mm}$ EPSS Steel locking screws.

\section{Mechanical testing}

The biomechanical characterisation of the fixation provided by both plate and nail constructs was assessed using cadaveric rabbit humeri from 12 skeletally mature rabbits of equivalent age and weight range as those included in the in vivo study. The humeri were divided into 2 groups ( $n=6$ per group): Group 1 received a plate and group 2 received a IM nail. After implanting the plates and nails, a transverse $0.45 \mathrm{~mm}$ osteotomy was created in the middle of the diaphysis (as per surgical procedure described below). Biomechanical testing was performed using a material testing system (MTS mini Bionix 858, MTS USA). Bones were first subject to three axial quasistatic ramp cycles up to $30 \mathrm{~N}$ compression at a rate of $1.5 \mathrm{~N} / \mathrm{s}$. Subsequently, the specimens underwent three torsional quasistatic ramp cycles up to $400 \mathrm{Nmm}$ at a rate of $20 \mathrm{Nmm} / \mathrm{s}$. Internal and external torque were applied in an alternating fashion, holding axial load at zero. After the third cycle, internal rotation was applied until construct failure applying the same angular rate. Parameters of interest were: axial stiffness, combined (between $400 \mathrm{Nmm}$ internal rotation and $400 \mathrm{Nmm}$ external rotation) and internal (between $800 \mathrm{Nmm}$ and $1000 \mathrm{Nmm}$ ) torsional stiffness, play around neutral zone, torque and angle at failure. Each construct was compared and normalised with the intact contralateral humerus from the same animal.

\section{In vivo study}

The in vivo study was approved by the ethical committee of the canton of Grisons in Switzerland (approval numbers 12/2012, 6/2013, 33/2012). All procedures were performed in an AAALAC (Association for Assessment and Accreditation of Laboratory Animal Care International) approved facility and according to Swiss animal protection law and regulations. Fifty nine skeletally mature specific pathogen free (SPF) female New Zealand White rabbits (Charles River, Germany) between 24 and 36 weeks of age and a mean body weight of $4.3 \pm 0.6 \mathrm{~kg}$ were finally included in this study.

\section{Surgical anaesthesia protocol}

The animals were sedated prior to surgery using a combination of $0.2 \mathrm{mg} / \mathrm{kg}$ Medetomidine (Domitor ${ }^{\circledR}$, Pfizer AG, Switzerland), $0.5 \mathrm{mg} / \mathrm{kg}$ Midazolam (Dormicum ${ }^{\circledR}$, Roche Pharma AG, Switzerland) and $0.005 \mathrm{mg} /$ kg Fentanyl (Sintenyl ${ }^{\circledR}$, Sintetica SA, Switzerland) intramuscularly. Anaesthesia was induced with Propofol (Propofol $^{\circledR} 1 \%$ MCT Fresenius, Fresenius Kabi AG,
Switzerland) intravenously to effect $(2-6 \mathrm{mg} / \mathrm{kg})$. The rabbits were intubated and anaesthesia was maintained with 1.5 to $2 \%$ Isoflurane (Isofluran Baxter ${ }^{\circledR}$, Baxter AG, Switzerland) in oxygen. Preemptive analgesia was achieved by $4 \mathrm{mg} / \mathrm{kg}$ Carprofene (Rimadyl ${ }^{\circledR}$, Pfizer AG, Switzerland) intravenously during surgical preparation and $0.01 \mathrm{mg} / \mathrm{kg}$ Fentanyl intravenously immediately before incision. Analgesia was repeated every 20 min thereafter during surgery. Post-operative analgesia consisted of Carprofene (Rimadyl ${ }^{\circledR}$, Pfizer AG, Switzerland) (4 mg/ $\mathrm{kg}$ ) subcutaneously once daily for 3 days, Buprenorphine (Temgesic ${ }^{\circledR}$, Reckitt Benckiser AG, Switzerland) (0.05 mg/ $\mathrm{kg}$ ) intramuscularly three times daily for 1 day and a Fentanyl patch (Fentanyl Mepha ${ }^{\circledR} 12 \mu \mathrm{g} / \mathrm{h}$, Mepha Pharma AG, Switzerland) which was applied to the skin during surgical preparation.

\section{Nailing procedure}

RISystem AG, Davos, Switzerland, provided all implants and instruments for the nailing procedure unless otherwise stated. The animals were placed in left lateral recumbency with the operated (right) limb up. The surgical side was clipped, washed with chlorhexidine-soap (Hibiscrub ${ }^{\circledR}$, CPS Cito Pharma Services, Switzerland) and wiped with alcohol (Softasept ${ }^{\circledR}$ N, B. Braun Medial AG; Switzerland) before being draped for aseptic surgery. A lateral incision was made over the proximal humerus with preparation down to its craniolateral surface for access to the medullary cavity. The access point was identified by fluoroscopy and created by drilling with a $2 \mathrm{~mm}$ drill bit. The entry hole and the medullary cavity were hand reamed to the length of the nail with a $3 \mathrm{~mm}$ reamer. After flushing the medullary cavity with isotonic fluid, the nail was passed and seated so the proximal end became flush with the entry point in the humerus. Placement was monitored by fluoroscopy. The incision was extended distally over the anticipated osteotomy site down to a point proximal to the anticipated most distal bolt hole and avoiding the radial nerve. The interlocking bolts were placed, from proximal to distal, by drilling a bicortical hole with a $1 \mathrm{~mm}$ drill bit and then over-drilling the cis cortex with a $1.1 \mathrm{~mm}$ drill bit. A $14 \mathrm{~mm}$ bolt was used for the proximal hole and $10 \mathrm{~mm}$ bolts were used for the 3 remaining holes. The bolts were inserted until the bolt was completely inserted through the nail or the insertion forces resulted in breakage of the conduit between the bolt and the handle. In all cases, interlocking bolts were considered to be firmly placed within the bone prior to closure of wounds. The osteotomy was created between the second and third interlocking bolt by means of a 0.45 mm Gigli saw. Sutures (\# 4-0 Monocryl, Ethicon, Belgium) were then preplaced through the surgical incision and only tightened after inoculation. Inoculation was performed by pipetting $34 \mu \mathrm{L}$ of the bacterial suspension directly over the osteotomy site (total number of bacteria added was measured and recorded, as described below). The skin was then finally closed (\# 5-0 Vicryl rapide, Ethicon, Belgium) in a continuous pattern.

\section{Plating procedure}

The rabbits were prepared for surgery as described above for the nailing procedure. A craniolateral skin incision was 
extended between the M. biceps brachii and M. brachii, cranial to the V. cephalica using blunt and sharp dissection to expose the lateral surface of the humerus. To protect the radial nerve, tissue handling and dissection in the caudal and distal area of the radius was reduced to a minimum. A stab incision was made between the M. brachii and the caput laterale of the $\mathrm{M}$. triceps to accommodate the most distal screw. The humerus was freed from surrounding tissue in the area of the intended osteotomy. The 7-hole locking plate was placed on the lateral humerus. The most proximal hole was drilled with a $1.8 \mathrm{~mm}$ drill bit and guide. The screw length was measured and a locking screw of appropriate length was placed (Synthes, $2.0 \mathrm{~mm}$ diameter). The remaining screws were placed in the same fashion. A full mid-diaphyseal osteotomy was created using a $0.45 \mathrm{~mm}$ Gigly saw (RISystem, Davos, Switzerland) halfway between the third and fourth screw, underneath the unused central screw hole. For the inoculated group, the sutures (\# 4-0 Monocryl) for the fascia were preplaced and fully closed after inoculation. Inoculation was performed by pipetting separate $34 \mu \mathrm{L}$ injections onto the central screw hole overlying the osteotomy and to the adjacent proximal and distal screw holes (total number of bacteria added was measured and recorded, as described below). Finally, a two-layer closure of fascia (\# 4-0 Monocryl) and skin (\# 5-0 Vicryl rapide) in a continuous pattern was performed.

\section{Animal welfare and observation}

All animals were screened prior to entry into the study and found to be healthy after a standard clinical examination. Approved animals were then allowed to acclimatise to their surroundings for two weeks prior to the start of the study. During this time, they were group housed with a $12 \mathrm{~h}$ dark/ $12 \mathrm{~h}$ light cycle, fed with hay, lettuce and supplemental feed for rabbits (Biomill, Switzerland). After surgery, the animals were single-housed. In the uninfected groups animals were group-housed 2 to 3 weeks after surgery. The inoculated animals were single housed for the whole duration of the study. For three days after surgery, each animal was checked at least twice daily by a veterinarian or an experienced animal caretaker. Thereafter, the rabbits were checked at least once a day for the rest of the study duration.

Blood samples were taken preoperatively, 3 days post-operative and weekly thereafter until the end of the observation period for white blood cell (WBC) count (Vet ABC, Scil animal care, Viernheim, Germany) and C-reactive protein (Rabbit CRP ELISA Kit, ICL Inc. Portland, Oregon, USA) in the inoculated groups. Weight was measured at surgery, after three days and weekly thereafter. Radiographs of the operated limb were taken in two planes after surgery and once a week thereafter for the rest of the study. Animal exclusion criteria were set at a weight loss exceeding $15 \%$ of the initial body weight within two weeks, local infection with severe lameness, persistent swelling and discharge, or signs of systemic infection such as fever, depression and anorexia. A small number of rabbits from all groups showed proprioceptive deficits on the operated leg after surgery. This was due to stress to the radial nerve during surgery and resolved usually within a week after surgery. Upon completion of the observation period, all animals were humanely euthanised using intravenously administered Pentobarbital (Esconarkon ${ }^{\circledR}$, Streuli Pharma AG, Switzerland).

\section{Bacterial inoculum preparation}

The inoculated S. aureus isolate (JAR060131) is a clinical isolate from an infected human hip prosthesis. The strain is a moderate biofilm former by the Stepanovic classification (Stepanovic et al., 2007) and possesses LukD/E leukocidin, gamma haemolysin, as well as staphylococcal enterotoxin b. The strain retains numerous adhesins including fibronectin, fibrinogen, laminin and elastin, although it does not contain the collagen or bone sialoprotein binding protein (Campoccia et al., 2008; Moriarty et al., 2009). The strain is broadly antibiotic sensitive, except for resistance to penicillin and is spa-type t084. The strain has been submitted to the Swiss Culture Collection, with accession number CCOS 890 . The bacterial inocula were individually prepared in Phosphate buffered saline solution (PBS, Sigma-Aldrich, Switzerland) for each surgery as previously described (Moriarty et al., 2010a). A dose response curve was created for each implant group, and was performed in a stage-wise manner to ensure full characterisation of the dose response curve but minimising the risk of exposing the rabbits to excessive inocula. The prepared inocula ranged from $6 \times 10^{3}$ colony forming units (CFU) to $6 \times 10^{6} \mathrm{CFU}$ for the plate surgeries, and from $6 \times 10^{2} \mathrm{CFU}$ to $6 \times 10^{6} \mathrm{CFU}$ for the nail surgeries. Quantitative culture of each inoculum was performed immediately after preparation to check the accuracy of the prepared inoculum and equivalence between the nail and plate inoculation procedures.

\section{Histology}

All animals from the uninoculated group and two infected animals from both the nailing and plating (10 and 4 weeks) groups (selected based on radiographic signs of infection) were processed for qualitative histological evaluation. The remaining rabbits in the inoculated groups were retained for quantitative bacteriology. A contact radiograph (full thickness) was taken using high-resolution technical film (D4 Structurix DW ETE, Agfa, Belgium) and a cabinet X-ray system (Model No. 43855A, Faxitron X-Ray Corporation, USA). After removal of the proximal and distal bones, the humeri underwent fixation with $70 \%$ ethanol for 4 weeks, with fresh ethanol changes weekly. Following fixation, samples were dehydrated through an ascending series of ethanol. They were then transferred to xylene, and finally infiltrated with and embedded in methylmethacrylate (MMA). The polymerised MMA blocks were trimmed using a butcher saw (Bizerba FK 22, Bizerba AG, Switzerland) prior to cutting with an annular diamond saw (Leitz 1600 saw microtome, Leica AG, Switzerland). The samples were glued with cyanoacrylate onto holders for sectioning. Serial sections of approximately $200 \mu \mathrm{m}$ thick were made, which were then submitted for contact radiography as described above. One section from each sample was selected, glued onto opaque plexiglass slides, ground and polished down to a final thickness of approximately $115 \mu \mathrm{m}$ and stained with Giemsa/Eosin. Histopathological analysis of all slides of all 
animals was performed using a light microscope (Olympus, Switzerland). Histological findings were described, wherever possible, according to distribution, severity and morphologic character. Healing in all groups was semiquantitatively scored on a 5-point scale, which evaluated osteotomy gap closure, callus size, and inflammation. The histopathological analysis included the hard and soft tissue local reaction with special emphasis on character and localisation of inflammatory changes and osteotomy healing. The score was developed specifically for this model by a veterinary pathologist.

\section{Bacteriology}

Bacteriological evaluations were only performed on animals included in the inoculation groups. The entire soft tissue surrounding the humerus was removed from the animals receiving the nail fixation, and the interlocking bolts and IM nail transferred to a single sterile receptacle containing PBS. For the animals receiving a plate fixation, the soft tissues immediately contacting the plate were removed using a sterile scalpel, and placed in a sterile receptacle containing PBS. The proximal screws, distal screws and the plate were then completely submerged in three separate sterile receptacles containing PBS.

The soft tissue samples (nail and plate groups) were roughly cut into approximately $0.5 \mathrm{~cm}$ pieces using a sterile scissors and homogenised using an Omni-TH hand-held homogeniser (LabForce AG, Switzerland) with a sterile Omni-tip plastic probes. The bone samples were roughly cut into small fragments no larger than $0.5 \mathrm{~cm}$ using a sterile luer and immediately homogenised using a Polytron PT3100 (Kinematica AG, Switzerland). The screws, plates and IM nails plus bolts were vortexed for $20 \mathrm{~s}$ followed by sonication for $3 \mathrm{~min}$ at $35 \mathrm{kHz}$ in an ultrasonicating water bath (Bandelin Sonorex Super 10P, Bandelin, Germany).

All homogenised tissue samples and sonicated implant samples were then immediately serially diluted in PBS and plated onto blood agar (BA) plates. BA plates were prepared using Blood agar base (Oxoid AG, Switzerland), containing $5 \%$ defibrinated horse blood. Agar plates were incubated at $37^{\circ} \mathrm{C}$ and colonies counted at 24 and $48 \mathrm{~h}$. The lower limit of detection (LLOD) for bacteria was $200 \mathrm{CFU} /$ bone fragment, $100 \mathrm{CFU} /$ soft tissue and $200 \mathrm{CFU} / \mathrm{IM}$ nail. All bacterial growth was evaluated by latex agglutination test for identification of infecting bacteria as $S$. aureus (Staphaurex Plus, Oxoid AG, Switzerland).

\section{Statistical analysis}

Results are presented as means of each group with standard error of the mean (s.e.m.). Results of biomechanical testing were tested for normal distribution by Shapiro Wilk test, and found to be greater than 0.05 (indicating normal distribution), therefore a student $t$-test was applied for statistical evaluation. A two way ANOVA was used to compare differences between infected and noninoculated groups with regards to clinical observations (CRP, Lymphocyte ratio and weight change). In all cases, significance was set at $* p<0.05$. Prism software was used for all statistical tests (GraphPad Software Inc., La Jolla, CA). In the infection study, a grouped sequential procedure was used, whereby the inoculum for each experimental phase was chosen based on results from all preceding phases as used for previous similar studies (Schlegel and Perren, 2006). The stepwise adjustment of the infectious doses allowed placement of increased number of animals at the dose levels at which the difference in infection rate is most evident (i.e. Infectious Dose 50, $\mathrm{ID}_{50}$ ) and minimising the number of animals at other doses. The calculation of the $\mathrm{ID}_{50}$ was based on cumulative infection rates according to the method described by Reed (Reed and Muench, 1938).

\section{Results}

Mechanical testing of constructs in cadaveric humeri Summary data of mechanical testing results is shown in Fig. 2. The cause of failure in the control (intact) groups under torsional loading was an oblique fracture in the bone, whereas the construct failure in both implant groups occurred by bone fracture through the screws or bolts, primarily in the distal fragment. The plate constructs failed at a higher torque than nail constructs $(p<0.05)$. No difference was observed between both groups of implants regarding axial stiffness, combined and internal torsional stiffness. There was a trend for significance regarding the play around neutral zone $(p=0.06)$.

\section{Healing in the non-inoculated nail model}

Nine rabbits were operated in the non-infected IM nail healing group. One rabbit had to be euthanised intraoperatively due to a fractured humerus after insertion of the nail. All other animals recovered fully from surgery. The time-point for euthanasia was set at the earliest point of assured radiographic evidence of healing. After 4 weeks, callus formation was evident on radiographs and all rabbits showed good weight bearing on the operated limb. After 10 weeks, all rabbits were fully load-bearing upon the operated leg and conventional radiographs displayed good callus formation and bridging between the proximal and distal diaphysis (Fig. 3).

\section{Healing in the non-inoculated plate model}

Ten rabbits were included in the non-inoculated-plate healing group. One rabbit had to be euthanised intraoperatively due to a fractured humerus. One rabbit was euthanised 4 days after surgery due to persistent lameness. All other animals tolerated the surgical procedure and survived the entire observation period. The time-point for euthanasia was set at the earliest point of radiographic evidence of complete healing. Callus formation was evident in radiographs of these rabbits after 4 weeks and all rabbits were at least partially weight bearing on the operated limb by this time. After 10 weeks, all rabbits were fully load-bearing upon the operated leg and contact radiographs displayed callus regression and bridging between the proximal and distal diaphysis (Fig. 3).

\section{Infection development}

A total of 25 rabbits were included in the inoculated nail group. All animals survived the surgical procedure, although four of the first five operated rabbits were euthanised early due to fracture of the humerus close to the osteotomy gap within 14 days. These animals were 


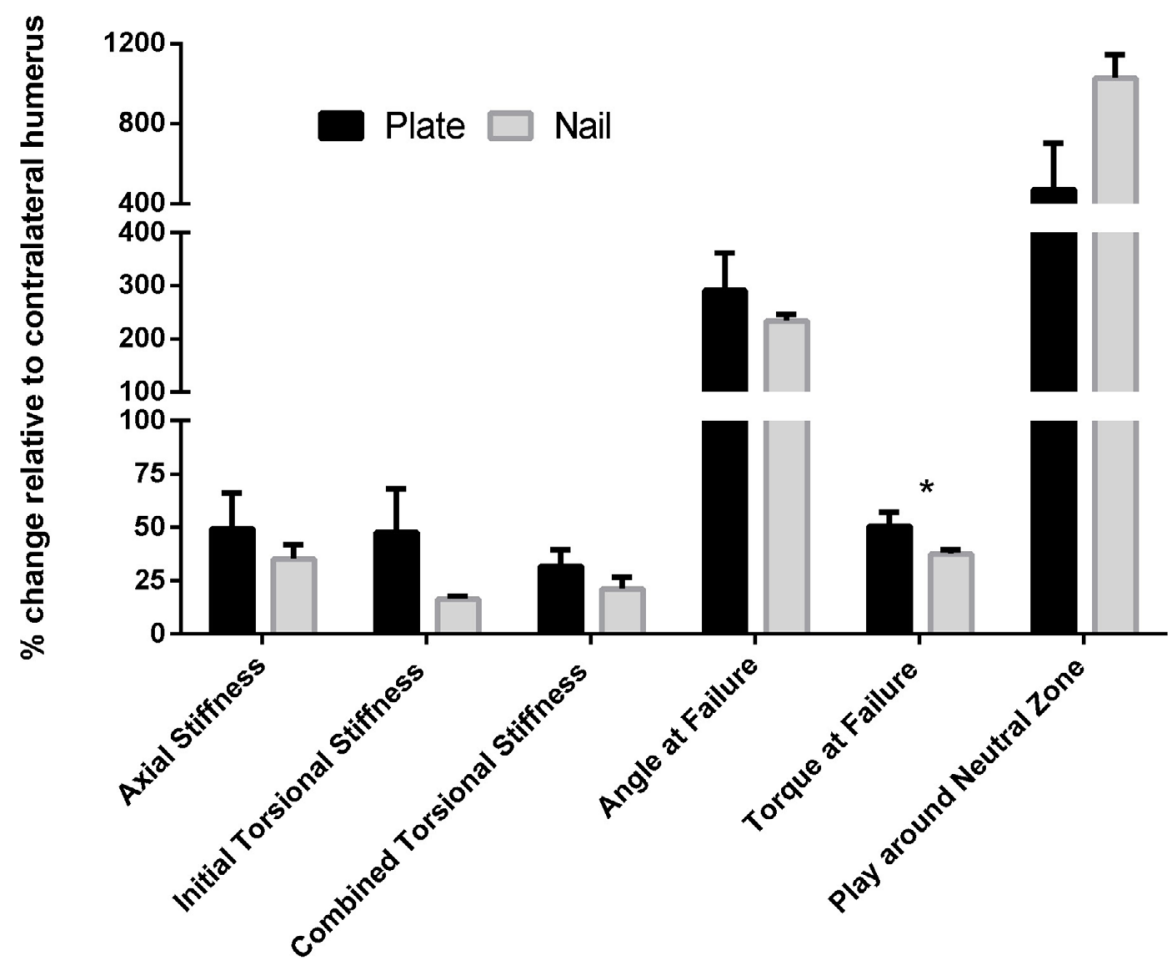

Fig 2. Overview of mechanical testing results for both plate and nail groups versus intact bones $\left(n=6\right.$ per group, $\left.{ }^{*} p<0.05\right)$.

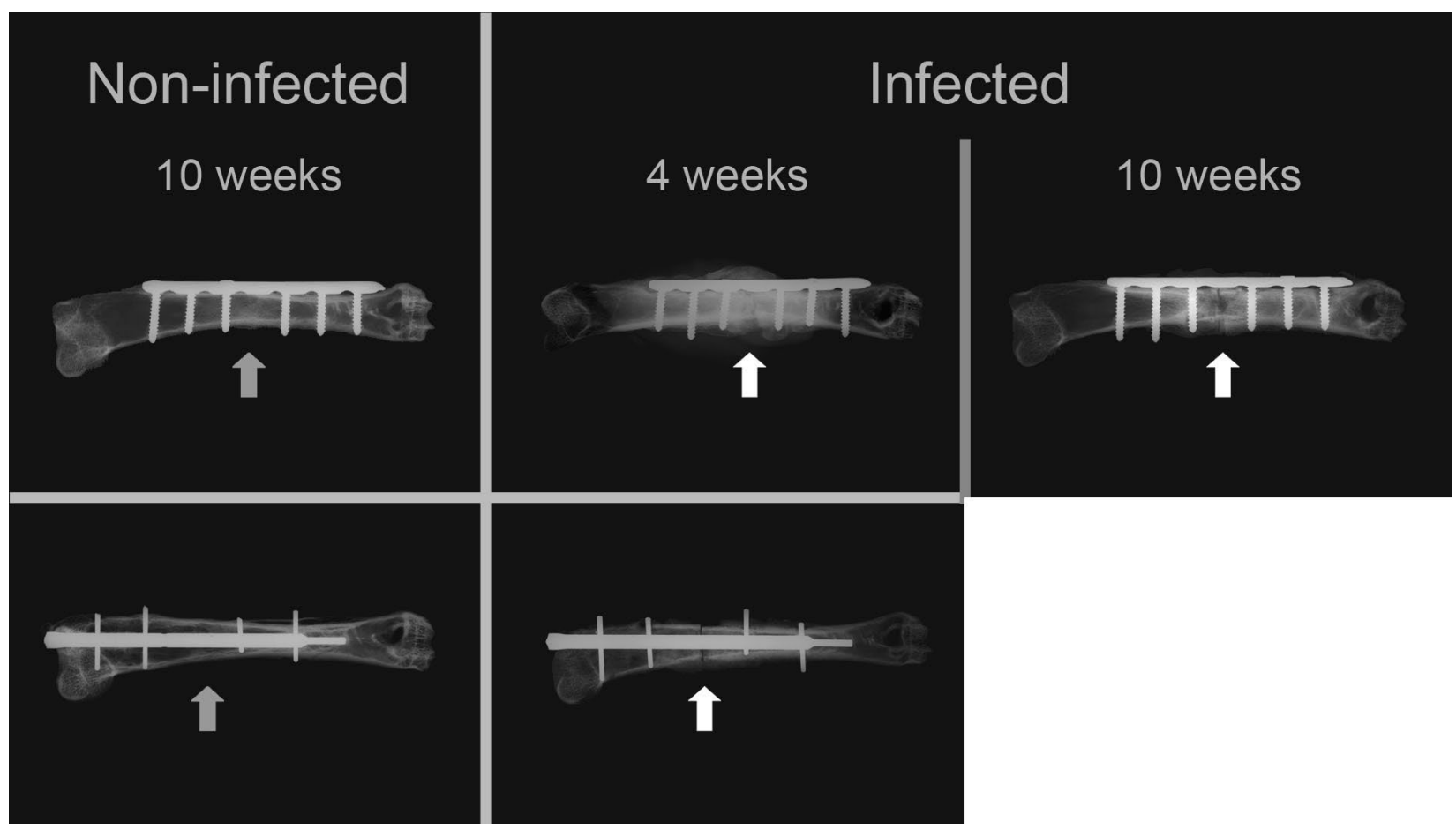

Fig. 3. Representative contact radiographs of rabbits in each group upon completion of the respective observation periods. Both plate and nail fixation lead to healing in the non-infected group at 10 weeks. In the infected animals at 4 weeks, the osteotomy gap is still visible in both nail and plate fixation, with periosteal reaction also observed in the plate group. In the long-term infection group at 10 weeks with plate fixation, the osteotomy gap is also still visible, although with reduced periosteal reaction compared to the 4 weeks group. Note: the third interlocking bolt in the nailing procedure is placed at an angle relative to the other bolts (described in Materials and Methods). 


\section{A: CFU Plate}

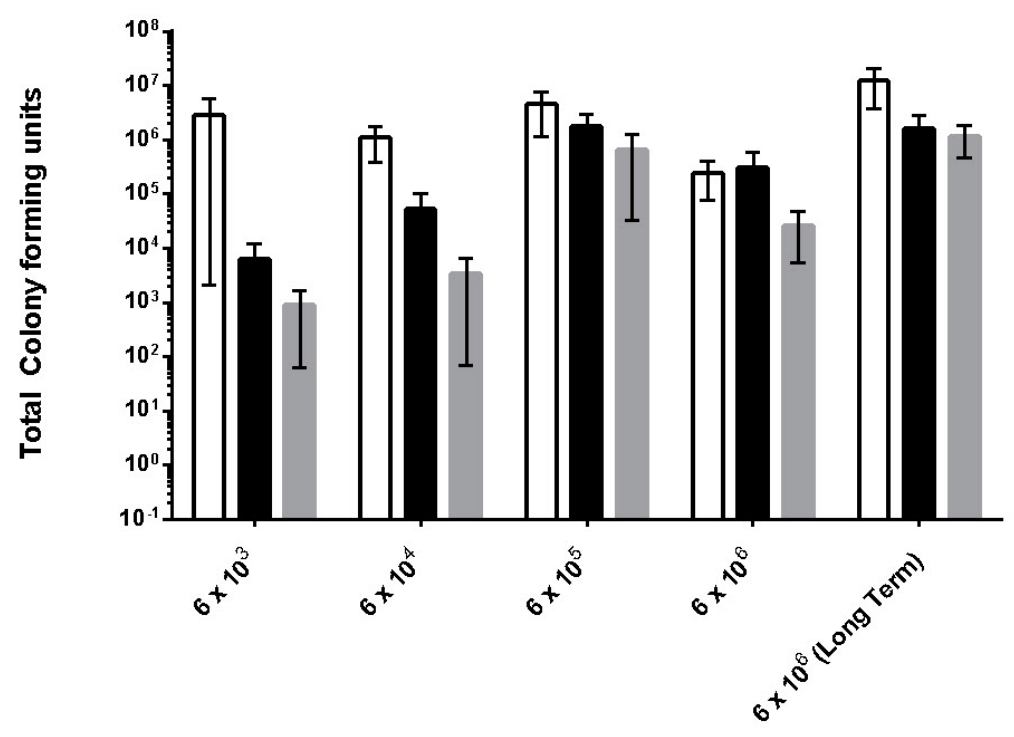

\section{B: CFU Nail}

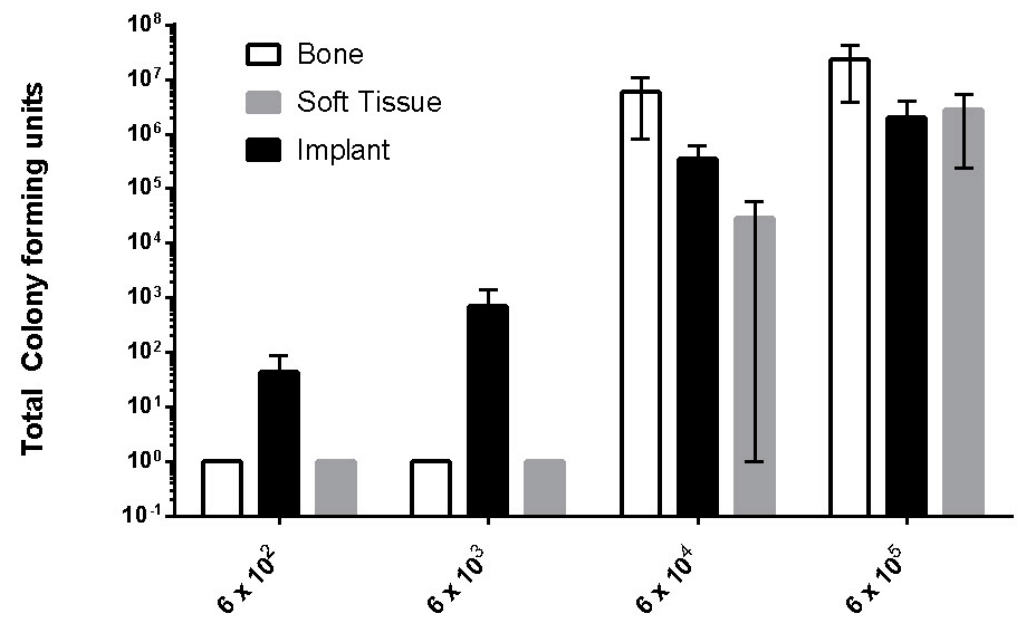

\section{C: Infection Rate}

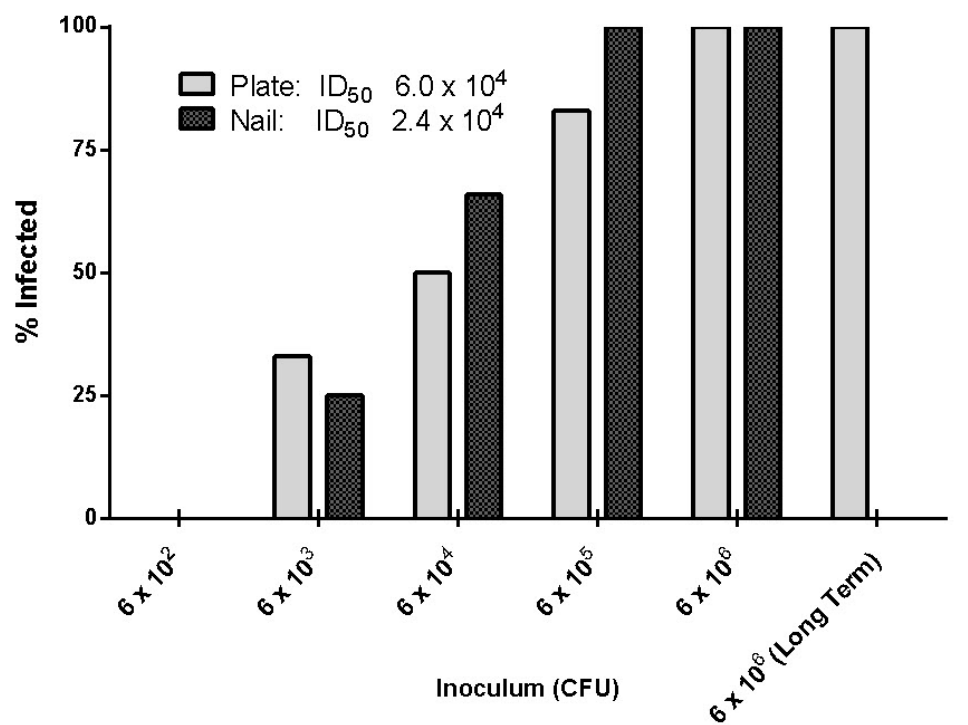

Fig. 4. Quantitative bacteriology from implant, bone and soft tissue from each infected rabbit in the inoculated group; A, plate group and B nail group (Note: non-infected animals not shown). C, Dose-response and $\mathrm{ID}_{50}$ for $S$. aureus inoculation in the plate (short and long term) and IM nail model. 
Plate

Nail

A: CRP
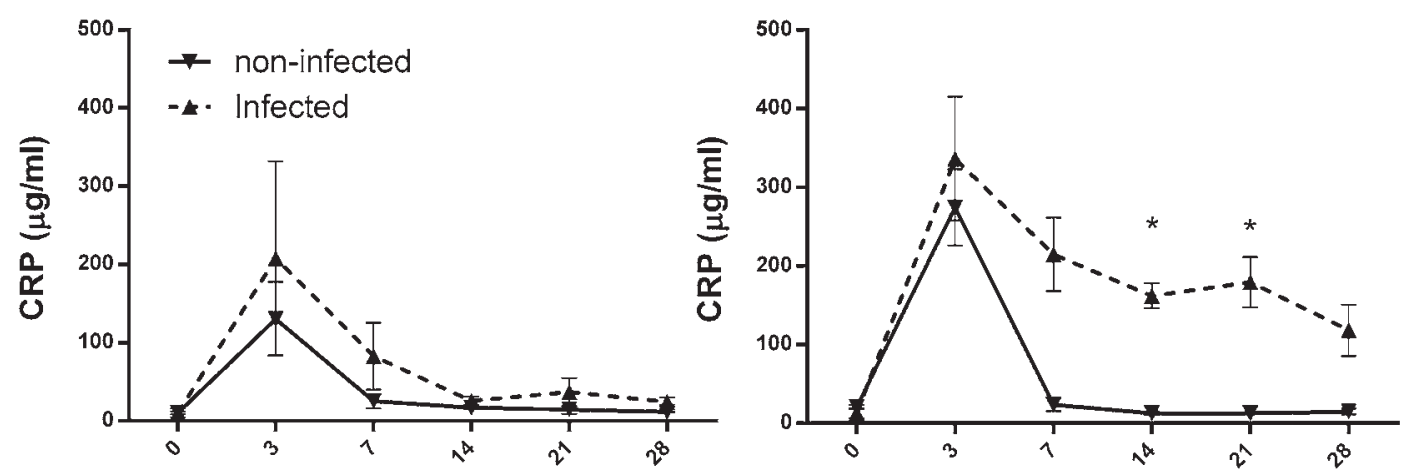

\section{B: Lymphocyte:granulocyte ratio}
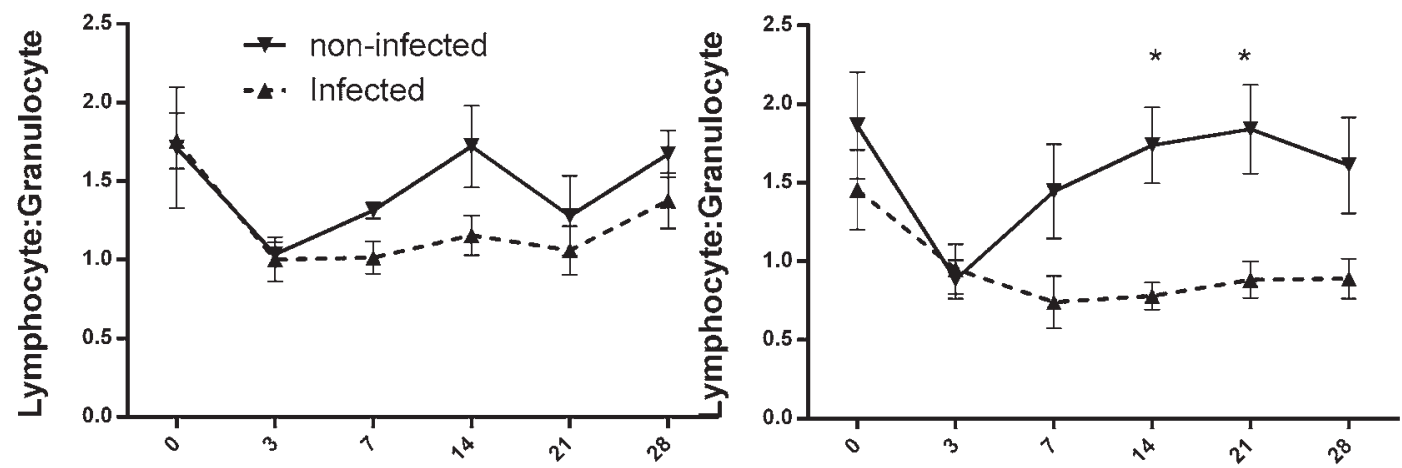

\section{C: Weight Change}
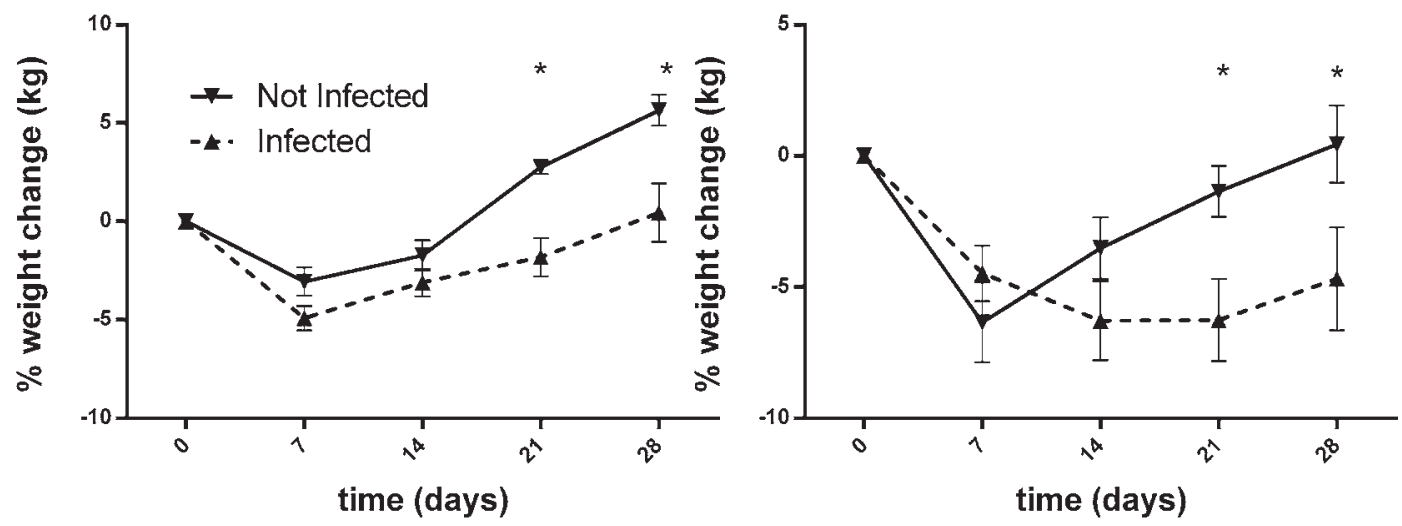

Fig. 5. Haematological and clinical observations of rabbits receiving a plate (left) and nail (right) in the infection study. A, CRP concentration; B, Ratio of lymphocytes to granulocytes; $\mathbf{C}$, Weight change from pre-operative weight. In all cases $n \geq 6$, and data resented as mean \pm s.e.m.

housed in a hutch with a raised platform as a form of housing enrichment, but are believed to have fractured the limb while jumping up to or down from the platform. The platform was then removed for all remaining animals. Another issue identified in these animals was that in three of four cases, the most proximal interlocking pin failed to fully engage in the trans cortex, which was identified at that time as a critical parameter for all subsequent surgeries. The remainder of the rabbits survived the entire observation period without any complications. Infection rate was assessed at five doses, in ten-fold increments commencing at a low dose of $6 \times 10^{2} \mathrm{CFU}$. Overall, 14 of the 21 included rabbits were eventually determined to be infected, with an increasing rate of infection with an increasing inoculum (Fig. 4). The ID $_{50}$ was calculated to be $2.4 \times 10^{4} \mathrm{CFU}$. In all cases, infected animals were culture positive, although animals receiving the lower inocula tended to yield a lower CFU count.

A total of 17 rabbits were included in the inoculated group receiving plating fixation. All animals survived the 


\section{Healing at 10 weeks}

Infection at 4 weeks
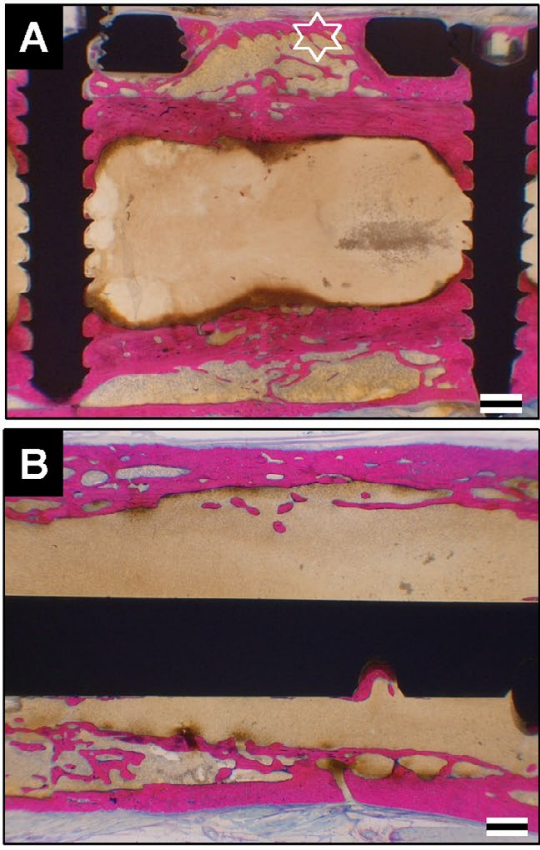
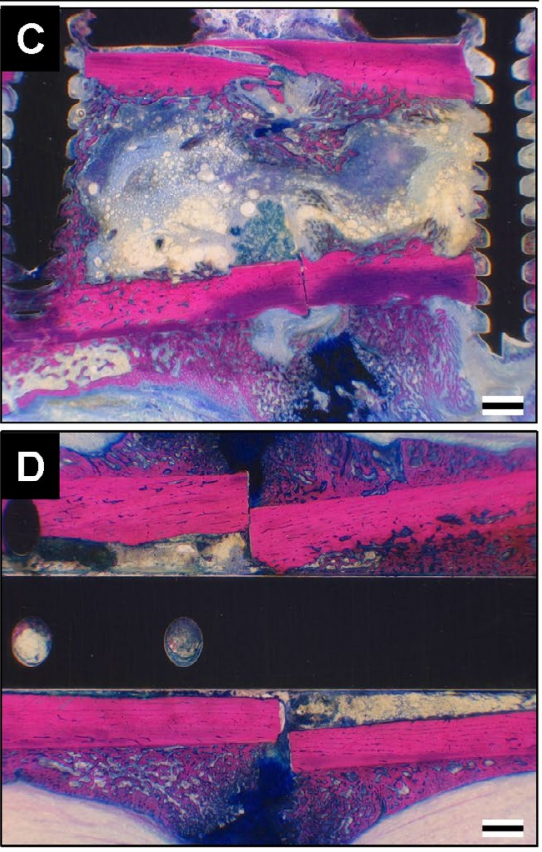

Infection at 10 weeks
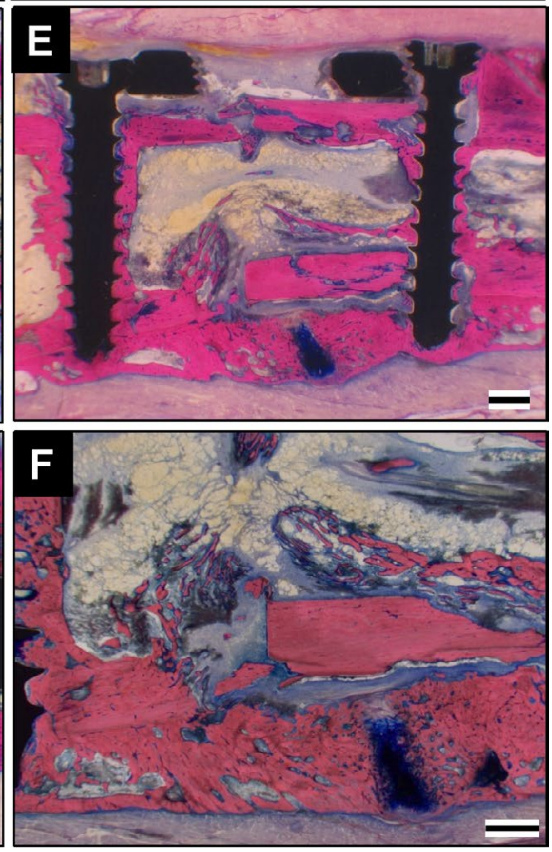

Fig. 6. Giemsa Eosin stained histological sections of osteotomy region in rabbits in the healing and infection studies. $\mathbf{A} \& \mathbf{B}$, non-infected (10 weeks post implantation) receiving a plate (A) or nail (B). Note new bone formation around the plate (star in $\mathbf{A}$ ) and complete osteotomy closure in both groups. Infected animals at four weeks $(\mathbf{C}$ and $\mathbf{D})$ do not display any signs of osteotomy healing in either group, with significant inflammation observed. A long-term infection group at ten weeks in the plate group $(\mathbf{E} \& \mathbf{F})$ reveal failure to heal at ten weeks with further areas of inflammation, osteolysis and fibrous encapsulation observed. All sections are stained with Giemsa Eosin, scale bar $=1 \mathrm{~mm}$.

Table 2. Mean histopathology score in each group in the healing study (median (range))*.

\begin{tabular}{|l|c|c|}
\hline Feature & Non-infected Plate 10 weeks & Non-infected Nail 10 weeks \\
\hline Osteotomy closure & $4(3-5)$ & $5(5-5)$ \\
\hline Callus size & $3(2-3)$ & $2(2-2)$ \\
\hline Periosteal Inflammation & $0(0-0)$ & $0(0-0)$ \\
\hline Medullary Inflammation & $0(0-0)$ & $0(0-2)$ \\
\hline
\end{tabular}

* Grade: 0 absent; 1 minimal; 2, slight; 3, moderate; 4, marked; 5, massive/complete.

surgical procedure and the four-week observation period. Infection rate was assessed at four doses, in ten-fold increments commencing at a low dose of $6 \times 10^{3} \mathrm{CFU}$. Overall, 11 of the 17 rabbits were eventually determined to be infected, with an increasing rate of infection with an increasing inoculum (Fig. 4). The $\mathrm{ID}_{50}$ was calculated to be $6.0 \times 10^{4} \mathrm{CFU}$. In all cases, infected animals displayed significant colonisation of the implant and bone infection, as well as some soft tissue infection (Fig. 4).

Weight, CRP and White Blood Cell (WBC) counts were recorded throughout the observation period and are outlined in Fig. 5. Weight loss was greater for infected animals than non-infected in both the nail and plate model. Total WBC did not differ between infected and non-infected animals for either the plate or the nail groups. The lymphocyte: granulocyte ratio revealed a greater difference between infected and non-infected animals in both models, though more markedly in the nail model (Fig. 5). Non-infected rabbits in the nail group displayed a higher postoperative peak in CRP than plated rabbits, however, in both plate and nail groups, the infected animals displayed a higher and more persistently elevated CRP.

\section{Histological evaluation}

In both non-inoculated groups, complete osteotomy closure was observed at 10 weeks, although the osteotomy closure was graded slightly higher in the nail group, where no visible remnants of the original osteotomy were observed (Fig. 6, Table 2). The plate fixation group resulted in periosteal osseous new bone formation around the plate, which is a common feature of metal internal fixation plates. As expected, the nail group displayed some endosteal bone formation and bony integration of the implant, which was much less apparent in the plate group. A low-grade cartilage formation was observed at some boltholes in the nail group, indicating some instability within the nail group. No such instability was observed at the osteotomy site. In the plate group, there were some indications of potential stress shielding, evidenced by a slight reduced thickness of the CIS cortex. 
The inoculated groups displayed no signs of osteotomy closure in either group at four weeks. Active inflammation was observed in both groups, indicative of a sub-acute to chronic infection (Fig. 6). The plate group tended to display slightly greater inflammation at the periosteum, whilst the nail group displayed a slightly more pronounced medullary inflammation. Furthermore, the inflammation of the surrounding soft issue in the plate group displayed purulent to necrotising inflammation, in contrast to the mixed-cellular inflammation in the medullary region of the nail group. The histological results in the infected group are provided for illustrative purposes and not submitted for semi-quantitative scoring.

\section{Discussion}

Rabbit models of implant related osteomyelitis have been indispensable in experimental research into antibiotic selection and dosage (Lazzarini et al., 2006), development of new antimicrobial devices (Schurman et al., 1978) and basic implant infection susceptibility (Moriarty et al., 2010b; Schlegel and Perren, 2006). In the specific context of post-traumatic implant related osteomyelitis, the biomechanical properties of the implant system used is widely considered to influence the risk and progression of infection. Many rabbit models of implant related osteomyelitis avoid the use of fracture models (Alt et al., 2006; Giavaresi et al., 2014; Reifenrath et al., 2014) due to the complexity required to create and fix a bone defect requiring stabilisation. Fracture or defect models have been described in smaller rodent models (Inzana et al., 2015; Kim et al., 2014; Penn-Barwell et al., 2014), and these smaller scale models are clearly advantageous in terms of cost and ease of handling. However, the rabbit retains an important space in the preclinical testing in implant related osteomyelitis. The small size of rodents may in fact be a limitation in cases where, for example, coatings may not be reliably applied to particularly small implants, or in cases where local antibiotic delivery vehicles may need to be less than a few millimetres in order to fit within defects, which also may be a challenge. Rabbit models on the other hand offer the opportunity to use human scale implants, as evidenced by the use of human LCPs in this study, and interventions and may be delivered in more controllable and realistic volumes. The rabbit, therefore, provides an option for testing at a level in between the smaller rodents, and the larger animal models.

We describe here the development of a biomechanically defined osteotomy model in the rabbit that may be fixed with either plating or nailing osteosynthesis. Both the locking plate and interlocked intramedullary nail allow complete healing of the rabbit humerus within 10 weeks. The time frame for healing in our model appears to be quite consistent with similar studies showing osteotomy healing at approximately similar time points for external fixation (Tobita et al., 2012) intramedullary nailing (Lowry et al., 1997), and plating fixations (Terjesen, 1984b; Wang et al., 2005) in rabbits. Only limited literature is available to compare performance of our implant constructs with relevant equivalents. A previously described interlocked nail for rabbit tibia has been biomechanically tested (LeCronier et al., 2012). However, this has not been tested in living animals to the best of our knowledge. Another study following an IM nail-type fixation in rabbit humerus showed some stress shielding was apparent when biomechanical evaluations were performed (Lowry et al., 1997), although the unlocked stainless steel pins used in that study make direct comparison with our interlocked model difficult. Previously described plate constructs have also indicated some stress shielding may be apparent (Terjesen, 1984b), similar to our present findings. Biomechanical evaluation of cadaveric, fixed humeri in this study revealed a trend for increased play around neutral and angular stability in the nail group, which may be a factor in some of the histologically observed differences, but actually reflect clinical reality in that plating tends to offer greater stability than nails in general. Further tests on healed bones would be of interest to support our findings. However, our in vivo healing study proves that both the IM nail and plate provide sufficient strength to allow complete healing of the transverse osteotomy within an expected timeframe.

As infection is a serious complication of fracture care, particularly open fractures, we further developed the model as an implant related osteomyelitis model. Towards this goal, we performed a dose response study in both models with a clinical $S$. aureus strain isolated from an infected joint arthroplasty. $S$. aureus is the most commonly isolated microorganism from post-traumatic implant related bone infections (Trampuz and Zimmerli, 2006), typically causing infection characterised by bacterial biofilm formation on the implanted hardware, osteolysis of adjacent bone, periosteal reaction and indicators of acute infection such as elevated CRP and white blood cell count. In these models, infection is characterised by retarded fracture healing, disordered new bone formation and a localised colonisation of the implant. As such, they replicate implant related osteomyelitis as encountered in clinical medicine. In all cases, the infection was localised to the implant, bone and adjacent soft tissues, without any systemic signs associated with sepsis. We chose a time-point of four weeks as it allows time for the infection to establish and illicit these characteristic features of infection, without burdening a large number of animals with an extended infection period. This is also quite clinically relevant as $S$. aureus is considered to cause primarily acute infection. In order to see the long-term effects of infection in the model, we observed infection in the plated group. We could clearly show that the infection is not self-limiting and that the bone cannot heal in the presence of infection in the plate group. The same result is expected in the nail model, in the presence of infection, within the normal timeframe of healing for a non-infected rabbit, although further studies would be required to confirm this belief.

In comparing the clinical and histological findings, the most striking differences between groups were perhaps the elevated CRP level in the nail group in comparison with the plate group. The nailing procedure may be considered more invasive, as significant amounts of bone marrow will be disturbed or removed during reaming, which is not part of the plating procedure. Human clinical studies comparing 
CRP in nailing and plating procedures are limited and scarce, although one clinical study has shown that CRP is highly elevated after reamed nailing in comparison with screw fixation (Garnavos et al., 2005). Interestingly, even when infected, the nailed rabbits had a higher CRP than infected plated rabbits, and a potential reason for this may be the observation in histological sections that a greater intramedullary inflammation was observed in nailed rabbits, versus a soft tissue inflammation in plated rabbits.

Whilst the aim of the present study was to establish two parallel models of plating and nailing osteosynthesis, with and without infection, the results do allow some comparison between the two fixation types. The clinical literature does not indicate any difference exists in infection rate between nailing and plating procedures, although only few trials have been performed specifically focussing on infection risk (Kurup et al., 2011; Ma et al., 2013). In the only previously described experimental study involving both nail and plate models of infection, Worlock et al. compared compression plating with a relatively less stable K-wire "IM nail" group (Worlock et al., 1994). That study revealed that the plate model displayed significantly more resistance to infection, and the authors hypothesised that this was due to the difference in stability of fixation constructs. It must be mentioned that results were achieved using compression plating, rather than the more modern locking plate concept, and compared them with unlocked nail constructs (K-wires). In the present study, where locked plates and interlocked nails were used, with less significant differences in mechanical performance, the infectious dose $50\left(\mathrm{ID}_{50}\right)$ was only slightly higher in the plate than in the nail group. Combined, these findings agree with the basic outcome of the Worlock study (Worlock et al., 1994) in that stability seems to have a significant influence on infection risk, but when the nail is a more accurate reflection of clinical practice (and differences in stability are minor), this difference is much reduced. In our study, the magnitude of the difference between plate and nail fixation is rather low, and certainly not suggested to be extrapolated to the clinical situation and implant selection for human patients.

The developed models and our characterisation do have a number of limitations. For example, soft tissue damage is a common feature of fracture care, particularly for open fractures, although in the present study, soft tissue damage was controlled, reproducible, and limited to the surgical incisions and placement of the implant. Dedicated soft tissue trauma studies have been performed for rabbits (Guthrie et al., 2014) and rats (Kalicke et al., 2003), revealing that soft tissue damage may be a significant risk factor for infection (Kalicke et al., 2003). Similarly, models have been described with blunt surgically induced fractures (rather than clean osteotomies) in rabbits (Ashhurst et al., 1982). In this study, our focus lies in deep implant related osteomyelitis, and thus additional soft tissue damage and subsequent infection would increase the complexity of the model and risk severe soft tissue infections that may limit the observation period of our model. Further limitations include the fact that the fracture is caused by an osteotomy rather than a true fracture. Initial cadaveric trials of a blunt force trauma failed to produce consistent fractures in rabbit bones, which are more brittle than for example human bones. In many cases, the fracture pattern generated in these cadaveric rabbit bones were fixable with either the nail or the plate, although perhaps not both, and furthermore, in some cases the fractures were too proximal to be reliably fixed with either implant. Therefore, the fracture model would unavoidably result in some selection bias in the implant used to fix fractures and an increase in euthanasia of animals due to inappropriate fracture patterns, not to mention increased burden upon the rabbit. Another issue for discussion is the means of inoculation of bacteria (directly into the wound in a saline injection). Many studies utilise a foreign body such as a collagen fleece or colonised implant in order to contain the dose and aid propagation of infection. In our study, where we wished to model a peri-operatively contaminated open fracture, we wish to inoculate within the surgical site and not provide any external influence on the progression of infection. Any "vehicle" used to control bacterial inoculation may influence progression of infection by serving as additional foreign bodies. One final issue is the performance of the staged- $\mathrm{ID}_{50}$ approach, whereby a dose-response study is performed in order to determine the lowest dose required to infect all animals, and to determine the $\mathrm{ID}_{50}$. Using this approach, the animals that do not get infected contribute to the calculation of the risk of infection, and thus are valuable results. However, in a conventional sense the process utilises a large number of animals at a range of doses, and therefore, relatively fewer animals at each dose. Future studies in our lab will look to use the minimal dose to achieve $100 \%$ infection, and will thus benefit from the dose-response study performed here.

A number of important animal welfare issues are noteworthy with regard to the described models. In terms of burden upon the animal, by choosing the humerus, rather than for example the femur or tibia, the rabbits have the opportunity to minimise weight-bearing on the operated limb, since they predominantly rest on their hind legs. Operating on the tibia would offer the opportunity to avoid the radial nerve issue present in the humerus model, however, we found the temporary proprioceptive deficit to be minimised with careful attention to nerve protection in the operating room. It is also important to note that pilot studies in the femur suffered from a high failure rate due to the greater forces applied in the rabbit hind limb. Another point is that this model, as described, resulted in no animal being excluded from this study due to septic complications, which was a stated goal of the model i.e. establish a localised infection, with low dropout rates and humane endpoints. Finally, it should be noted that the nailing procedure is the more complex procedure, and should be done by an experienced and/or trained surgeon to minimise post-operative exclusion rates.

As a further development of the model, it would be interesting to follow the infection as it progresses to a more chronic phase in the nail group. Similarly, it would be interesting to compare antibiotic treatment regimens in this model, both prophylactic and therapeutic. Currently, we see this model as particularly useful for observing the effects of various prophylactic strategies including local delivery vehicles and novel anti-biofilm agents. The model 
would also be particularly suitable for investigations into early treatment options. For example, should therapy begin before the critical first three-weeks, as clinical teaching indicates, antibiotic therapy may be successful (Zimmerli and Trampuz, 2013). Such antibiotic interventions were not attempted in the process of developing this model as we felt the outcome would be highly specific to a particular treatment regimen, and shall be considered for future separate studies. A second development for the model would be to include implant removal and or suppressive antibiotic therapy to monitor bone healing after these two interventions. Rabbit studies have previously shown that bone healing may continue after plate removal (Terjesen, 1984a; Terjesen, 1984b) and so it would be interesting to monitor further bone changes after implant removal in this model. Implant removal would theoretically be a simple procedure for the plate, which offers direct access to the screws through a thin layer of soft tissue. Nail removal on the other hand, would require an implant redesign as the nail itself and interlocking bolts were designed for this study and not yet designed for implant removal. A final option, also to be considered, would be the development of gap or bone defect models. Both the plate and the nail have the possibility to accommodate a larger gap, which may be particularly suitable for example in a debridement and retention experiment, or even treatment of infection by the Masquelet technique (Masquelet et al., 2000). Such studies are highly clinically relevant and achievable with the current model.

\section{Conclusion}

We have successfully developed a rabbit model that incorporates a humeral osteotomy that enables fixation with two implant systems i.e. locking plating and interlocking IM nail fixation. The model is a significant advance over current rabbit models, which have neglected the fracturehealing component, particularly considering the critical role played by biomechanics on bone healing and infection. Inclusion of a clinically realistic fracture fixation allows preclinical testing of basic science concepts or translation of novel technologies in a model tailored to post-traumatic osteosynthesis. The model is suitable for further studies, particularly those looking into delayed union due to infection, or interventions tailored towards posttraumatic osteomyelitis.

\section{Disclosures}

The authors have no disclosures relative to the presented work.

\section{Acknowledgements}

This work was funded by AOTrauma as part of the Clinical Priority Program Bone Infection (Grant number AR2013_03). Dirk Nehrbass, Abhay Gahukamble, Ahmed Seif El Din, Cameron Lutton, Tanja Schmid, Philipp Zerbe, Iris Keller, Sandra Thoeny, Nora Goudsouzian, all from AO Research Institute Davos, are acknowledged for their expert assistance in the design, performance and discussion of the presented work. Romano Matthys and Reto Nützi from RiSystem AG Davos, Switzerland are thanked for their role in the design and manufacture of the IM nails.

\section{References}

Alt V, Bitschnau A, Osterling J, Sewing A, Meyer C, Kraus R, Meissner SA, Wenisch S, Domann E, Schnettler R (2006) The effects of combined gentamicin-hydroxyapatite coating for cementless joint prostheses on the reduction of infection rates in a rabbit infection prophylaxis model. Biomaterials 27: 4627-4634.

Ashhurst DE, Hogg J, Perren SM (1982) A method for making reproducible experimental fractures of the rabbit tibia. Injury 14: 236-242.

Campoccia D, Montanaro L, Moriarty TF, Richards RG, Ravaioli S, Arciola CR (2008) The selection of appropriate bacterial strains in preclinical evaluation of infectionresistant biomaterials. Int J Artif Organs 31: 841-847.

Claes L, Eckert-Hubner K, Augat P (2002) The effect of mechanical stability on local vascularization and tissue differentiation in callus healing. Journal of Orthopaedic Research 20: 1099-1105.

Fei J, Yu HJ, Pan CJ, Zhao CH, Zhou YG, Wang Y (2010) Efficacy of a norvancomycin-loaded, PDLLAcoated plate in preventing early infection of rabbit tibia fracture. Orthopedics 33: doi: 10.3928/0147744720100329-06.

Gahukamble AD, McDowell A, Post V, Salavarrieta Varela J, Rochford ET, Richards RG, Patrick S, Moriarty TF (2014) Propionibacterium acnes and Staphylococcus lugdunensis cause pyogenic osteomyelitis in an intramedullary nail model in rabbits. J Clin Microbiol 52: 1595-1606.

Garnavos C, Xirou ST, Nikolatos A, Kanakaris N, Tzortzi P, Balbouzis T, Papavasiliou N (2005) Alteration of body temperature, erythrocyte sedimentation rate, and C-reactive protein after reamed intramedullary nailing: a prospective study. J Orthop Trauma 19: 323-328.

Giavaresi G, Meani E, Sartori M, Ferrari A, Bellini D, Sacchetta AC, Meraner J, Sambri A, Vocale C, Sambri V, Fini M, Romano CL (2014) Efficacy of antibacterial-loaded coating in an in vivo model of acutely highly contaminated implant. Int Orthop 38: 1505-1512.

Guthrie HC, Martin KR, Taylor C, Spear AM, Whiting R, Macildowie S, Clasper JC, Watts SA (2014) A preclinical evaluation of silver, iodine and Manuka honey based dressings in a model of traumatic extremity wounds contaminated with Staphylococcus aureus. Injury 45: 11711178.

Hankemeier S, Grassel S, Plenz G, Spiegel HU, Bruckner P, Probst A (2001) Alteration of fracture stability influences chondrogenesis, osteogenesis and immigration of macrophages. J Orthop Res 19: 531-538.

Inzana JA, Schwarz EM, Kates SL, Awad HA (2015) A novel murine model of established Staphylococcal bone infection in the presence of a fracture fixation plate to study therapies utilizing antibiotic-laden spacers after revision surgery. Bone 72: 128-136. 
Kalicke T, Schlegel U, Printzen G, Schneider E, Muhr G, Arens S (2003) Influence of a standardized closed soft tissue trauma on resistance to local infection. An experimental study in rats. J Orthop Res 21: 373-378.

Kim S, Bedigrew K, Guda T, Maloney WJ, Park S, Wenke JC, Yang YP (2014) Novel osteoinductive photocross-linkable chitosan-lactide-fibrinogen hydrogels enhance bone regeneration in critical size segmental bone defects. Acta Biomater 10: 5021-5033.

Kurup H, Hossain M, Andrew JG (2011) Dynamic compression plating versus locked intramedullary nailing for humeral shaft fractures in adults. Cochrane Database Syst Rev: 15: doi: 10.1002/14651858. CD005959.pub2.

Lazzarini L, Overgaard KA, Conti E, Shirtliff ME (2006) Experimental osteomyelitis: what have we learned from animal studies about the systemic treatment of osteomyelitis? J Chemother 18: 451-460.

LeCronier DJ, Papakonstantinou JS, Gheevarughese V, Beran CD, Walter NE, Atkinson PJ (2012) Development of an interlocked nail for segmental defects in the rabbit tibia. Proc Inst Mech Eng H 226: 330-336.

Lowry KJ, Hamson KR, Bear L, Peng YB, Calaluce R, Evans ML, Anglen JO, Allen WC (1997) Polycaprolactone/ glass bioabsorbable implant in a rabbit humerus fracture model. J Biomed Mater Res 36: 536-541.

Ma J, Xing D, Ma X, Gao F, Wei Q, Jia H, Feng R, Yu J, Wang J (2013) Intramedullary nail versus dynamic compression plate fixation in treating humeral shaft fractures: grading the evidence through a meta-analysis. PLoS One 8: e82075.

Masquelet AC, Fitoussi F, Begue T, Muller GP (2000) [Reconstruction of the long bones by the induced membrane and spongy autograft]. Ann Chir Plast Esthet 45: 346-353.

Metsemakers WJ, Emanuel N, Cohen O, Reichart M, Potapova I, Schmid T, Segal D, Riool M, Kwakman PH, de Boer L, de Breij A, Nibbering PH, Richards RG, Zaat SA, Moriarty TF (2015) A doxycycline-loaded polymerlipid encapsulation matrix coating for the prevention of implant-related osteomyelitis due to doxycycline-resistant methicillin-resistant Staphylococcus aureus. J Control Release 209: 47-56.

Moojen DJ, Vogely HC, Fleer A, Nikkels PG, Higham PA, Verbout AJ, Castelein RM, Dhert WJ (2009) Prophylaxis of infection and effects on osseointegration using a tobramycin-periapatite coating on titanium implants - an experimental study in the rabbit. J Orthop Res 27: 710-716.

Moriarty TF, Campoccia D, Nees SK, Boure LP, Richards RG (2010a) In vivo evaluation of the effect of intramedullary nail microtopography on the development of local infection in rabbits. Int J Artif Organs 33: 667-675.

Moriarty TF, Debefve L, Boure L, Campoccia D, Schlegel U, Richards RG (2009) Influence of material and microtopography on the development of local infection in vivo: experimental investigation in rabbits. Int $\mathrm{J}$ Artif Organs 32: 663-670.

Moriarty TF, Schlegel U, Perren S, Richards RG (2010b) Infection in fracture fixation: can we influence infection rates through implant design? J Mater Sci Mater Med 21: 1031-1035.

Norden CW (1970) Experimental osteomyelitis. I. A description of the model. J Infect Dis 122: 410-418.

Odekerken JC, Walenkamp GH, Brans BT, Welting TJ, Arts JJ (2014) The longitudinal assessment of osteomyelitis development by molecular imaging in a rabbit model. Biomed Res Int 2014: 424652.

Penn-Barwell JG, Rand BC, Brown KV, Wenke JC (2014) A versatile model of open-fracture infection : a contaminated segmental rat femur defect. Bone Joint Res 3: 187-192.

Perren SM (2002) Evolution of the internal fixation of long bone fractures. The scientific basis of biological internal fixation: choosing a new balance between stability and biology. J Bone Joint Surg Br 84: 1093-1110.

Reed LJ, Muench H (1938) A simple method of estimating fifty percent endpoints. American Journal of Epidemiology 27: 493-497.

Reifenrath J, Angrisani N, Lalk M, Besdo S (2014) Replacement, refinement, and reduction: Necessity of standardization and computational models for long bone fracture repair in animals. J Biomed Mater Res A 102: 2884-2900.

Reizner W, Hunter JG, O’Malley NT, Southgate RD, Schwarz EM, Kates SL (2014) A systematic review of animal models for Staphylococcus aureus osteomyelitis. Eur Cell Mater 27: 196-212.

Schlegel U, Perren SM (2006) Surgical aspects of infection involving osteosynthesis implants: implant design and resistance to local infection. Injury 37 Suppl 2: S67-73.

Schurman DJ, Trindade C, Hirshman HP, Moser K, Kajiyama G, Stevens P (1978) Antibiotic-acrylic bone cement composites. Studies of gentamicin and Palacos. J Bone Joint Surg Am 60: 978-984.

Smeltzer MS, Thomas JR, Hickmon SG, Skinner RA, Nelson CL, Griffith D, Parr TR, Jr., Evans RP (1997) Characterization of a rabbit model of staphylococcal osteomyelitis. J Orthop Res 15: 414-421.

Stepanovic S, Vukovic D, Hola V, Di Bonaventura G, Djukic S, Cirkovic I, Ruzicka F (2007) Quantification of biofilm in microtiter plates: overview of testing conditions and practical recommendations for assessment of biofilm production by staphylococci. APMIS 115: 891-899.

Stewart S, Barr S, Engiles J, Hickok NJ, Shapiro IM, Richardson DW, Parvizi J, Schaer TP (2012) Vancomycinmodified implant surface inhibits biofilm formation and supports bone-healing in an infected osteotomy model in sheep: a proof-of-concept study. J Bone Joint Surg Am 94: 1406-1415.

Terjesen T (1984a) Bone healing after metal plate fixation and external fixation of the osteotomized rabbit tibia. Acta Orthop Scand 55: 69-77.

Terjesen T (1984b) Plate fixation of tibial fractures in the rabbit. Correlation of bone strength with duration of fixation. Acta Orthop Scand 55: 452-456.

Tobita K, Ohnishi I, Matsumoto T, Ohashi S, Bessho M, Kaneko M, Nakamura K (2012) Measurement of mechanical properties on gap healing in a rabbit osteotomy 
model until the remodeling stage. Clin Biomech (Bristol, Avon) 27: 99-104.

Trampuz A, Zimmerli W (2006) Diagnosis and treatment of infections associated with fracture-fixation devices. Injury 37 Suppl 2: S59-66.

Wang XP, Zhang XL, Li ZG, Yu XG (2005) A first order system model of fracture healing. J Zhejiang Univ Sci B 6: 926-930.

Worlock P, Slack R, Harvey L, Mawhinney R (1994) The prevention of infection in open fractures: an experimental study of the effect of fracture stability. Injury 25: 31-38.

Zimmerli W, TrampuzA(2013) Biomaterial-Associated Infection: A Perspective from the Clinic. In: Biomaterials Associated Infection (Moriarty TF, Zaat SAJ, Busscher HJ, eds), Springer New York, pp 3-24.

\section{Discussion with Reviewers}

Thorsten Guehring: This model is very versatile and will undoubtedly be used to understand how infection reduces healing and for evaluating strategies or therapies to reduce infection. The authors state that future studies will use the minimal dose to achieve $100 \%$ infection. For some applications, such as looking at anti-infection strategies, this may not be the best strategy. Clinical infection rates vary greatly but are generally never over $50 \%$, with 5-20\% being more common. A bioburden challenge that produces $100 \%$ infection rate may be too challenging for some of the therapies that will be evaluated. What are the authors' thoughts on balancing reproducible infection but use of clinically-relevant levels of inoculum?

Authors: In our study, we see that we achieve an infection rate of approximately $25 \%$ with an inoculum of $6 \times 10 \mathrm{e} 3$ CFU and approximately $50 \%$ infection rate at $6 \times 10 \mathrm{e} 4$ CFU. Although it is not known what initial bacterial burden may be required to cause an infection in human patients, the actual numbers of bacteria alone will not be the single determining factor. Indeed the clinical literature proves that highly contaminated open fractures can receive adequate protection from infection in a proportion of cases. The general health status of the patient and the surgical procedure (duration and extent of tissue damage) also play a role. Nevertheless, the reviewer raises an interesting point and we feel that in developing an animal model, we aim to standardise all factors that may influence infection risk. As such, we work with healthy young animals that do not have any established risk factors for infection. This enables us to have uniform outcome measures, which will be particularly useful in future interventional studies where reduction in infection risk will be an outcome. With an infection rate of only $20 \%$ the number of animals to be used for such investigation would be at least 5 times higher. In our opinion, this approach would be queried by many, if not all, animal care and use committees.

With respect to the contention that higher inocula may lead to failure of potentially efficacious antimicrobial treatments, we would point out that the literature provides many examples of antibiotic therapies that successfully prevent infection in challenging models. If conventional antibiotic therapies are efficacious against relatively high inocula, then this should be the minimal standard expected of any novel intervention. If an antibiotic containing intervention (e.g. coating) will only work at lower bacterial challenges, it is unclear how this would translate into clinical practice. Would the coating fail in those patients with higher bacterial burden? These same patients are the ones most in need of protection.

Thorsten Guehring: How can the findings of this study be interpreted, given the underpowered nature of the study? Authors: We would like to highlight that the healing study was performed with 8 animals per group, with consistent results and thus we are confident the model is reproducible. The aim of the infection study was to perform a dose response curve, aimed at determining the $\mathrm{ID}_{50}$ and inoculum required to achieve $100 \%$ infection rate. As such, we have a group size of 6 at the crucial "middle" doses. This ID $_{50}$ approach is a somewhat less often used approach, and our protocol is in agreement with previous studies using the same approach e.g. Kälicke et al. (2006).

Thorsten Guehring: What is the effect of the inability to contain the inoculum during surgery?

Authors: We believe the inoculation of a clinical wound to be a complex, unpredictable feature of infection. Our models reflect many of the most important features, including a bone fracture/osteotomy, implants and surgical incisions. We believe the lack of containment of the inoculum to be another step towards the clinical reality. Of course, this will introduce some variation, and would be a drawback perhaps for studies where an infection must be created in all animals, regardless of the method (i.e. in a treatment study). However, our study was designed to determine the risk of infection, and as such, we believe our approach is preferable where the animals that clear the infection are as valuable as those developing infection.

Reviewer: Why was a 4-week model chosen for the infection part of the in vivo study whereas most animal models use a 6 weeks follow-up window?

Authors: As far as the authors are aware, there is a wide range of euthanasia time-points used for bone infection studies in animals. We believe that the time-point for euthanasia should be set based upon clinically dictated time-points that are relevant to each study. We chose our time points for the healing study (10 weeks) and infection study (4 weeks) based on the expectation that osteotomy healing should be complete at 10 weeks, and an infection will have firmly established by four weeks. Continuing the infection for a further 2 (or 6) weeks would not strengthen the contention that these animals are infected: we show there is a significant bacterial colonisation of the implant (biofilm), which is a hallmark of established infection. Extending this time point may be beneficial if our aim were to develop a model for non-union due to infection. However, our study primarily aimed at developing a model of $S$. aureus infection, which is typically an agent of acute infection. 
Reviewer: A positive control group with antibiotics showing that the defect can heal completely was not incorporated into the experimental design. The authors should explain why.

Authors: First of all, for clarity, we completely agree that we need to prove that the osteotomy can heal in the absence of infection, otherwise the model would not replicate a proper fracture fixation. We would like to point out that this was in fact proven in the separate healing study included in the manuscript, which clearly shows osteotomy healing at 10 weeks.

The addition of an antibiotic treated group (plus bacterial contamination) would be an avenue for further research in this model. It would show (in an antibiotic agent and dosage dependent manner) how the model reacts to treatment or prophylaxis against infection. Whilst we agree this would be an additional validation, we believe any antibiotic treatment would be specific to that treatment regimen and would raise a number of subsequent questions: e.g. would we choose a regimen based on human medicine, or a regimen based on veterinary medicine? Do we use antibiotics recommended in a dose-adjusted manner in animals based on human pharmacokinetics? Do we use a strain of $S$. aureus that is resistant to first line antibiotics or sensitive? Do we administer a preventative regimen or a therapeutic regimen?

From experience, we are aware that rabbits do not tolerate many antibiotics routinely administered to humans. Also, there are somewhat varying guidelines for treatment, and it may be that our treatment choice does not match guidelines in every jurisdiction. We do intend to determine the response to antibiotic treatment in these models at some point, but these would be tailored studies with specific questions in mind e.g. selection and timing of prophylaxis; selection and timing of treatment; implant removal and local antibiotic depots etc., etc..

Reviewer: Mechanical testing in operated animals is not performed for the inoculated (infected) animals. The authors should explain why this was not done.

Authors: For clarity, the mechanical testing was done on cadaveric humeri after osteotomy as a prelude to in vivo testing. Mechanical testing of constructs at euthanasia was not done for either the healing or the infection groups.

The purpose of the mechanical test was to describe the biomechanical properties of both implant groups, prior to placement in the animal. It would be interesting to measure mechanical strength as a measure of bone healing in both groups at the appropriate euthanasia time points. In this study, we opted to illustrate osteotomy healing (or lack thereof) histologically, which can clearly show the bone formation between osteotomy ends. Mechanical testing would provide a clear measure of bone strength, but would not allow histological evaluation of the bone due to the destructive nature of the test. Furthermore, the implants would need to be removed prior to testing, and currently the IM nail we use would be extremely challenging to remove from the bone without significant collateral damage to nearby bone. We feel opting for histological visualisation was therefore a reasonable decision for our study.

Volker Alt: Why did the authors not include biomechanical testing of infected bones? This would be of great relevance. Authors: As mentioned above, whilst we agree that biomechanical testing of infected bones would be of interest, we preferred to illustrate bone healing histologically. From the histology, we can clearly see the osteotomy has not healed in the infected group at four weeks for either group, and now with the additional data we show this also does not occur at 10 weeks for infected plates. Mechanical testing would provide additional supportive data, although the visual proof we feel is more compelling.

Volker Alt: As I understand, the authors first did the bone Fixation (with nail or plate) and then the osteotomy? By doing so the nailing or plating may have affected the osteotomy. Why not first do the osteotomy and then the fixation?

Authors: Post-operative radiographs clearly show a good reduction of the osteotomy and displacement was rare and, if present, very minor and did not influence healing. Performing the osteotomy first would be a logical step towards clinical reality. However, from the perspective of developing an experimental and reproducible model, the placement of the implants first minimises the soft tissue damage, significantly reduces surgical time, improves fracture reduction and does not compromise the inoculation protocol.

\section{Additional Reference}

Kälicke T, Schierholz J, Schlegel U, Frangen TM, Köller M, Printzen G, Seybold D, Klöckner S, Muhr G, Arens S (2006) Effect on infection resistance of a local antiseptic and antibiotic coating on osteosynthesis implants: an in vitro and in vivo study. J Orthop Res 24: 1622-1640.

Editor's Note: Scientific Editor in charge of the paper: Joost de Bruijn. 